\section{Labio leporino en la terminología biomédica}

\section{Cleft lip in biomedical terminology}

Sr. Editor: Hemos leído con gran interés el artículo publicado recientemente por Nazer et al. ${ }^{1}$ titulado "38 Años de vigilancia epidemiológica de labio leporino y paladar hendido en la maternidad del Hospital Clínico de la Universidad de Chile", en donde se reporta una vasta experiencia como miembro del ECLAMC (Estudio Colaborativo Latino Americano de Malformaciones Congénitas), llevando un registro de todos los recién nacidos vivos y mortinatos que presentan una o más anomalías congénitas, en la Maternidad del Hospital Clínico de la Universidad de Chile.

Sin embargo, nos ha llamado la atención en este artículo, así como en general en la literatura científica biomédica, la utilización del término "labio leporino", el cual creemos obedece a una denominación que debería dejar de ser utilizada. Esto debido a que el término "leporino" según la Real Academia Española (RAE) ${ }^{2}$ deriva del latín leporinnus, que tiene como significado "Perteneciente o relativo a la liebre".

Este término ha sido adscrito a los pacientes que padecen el defecto congénito consistente en una hendidura o separación del labio superior, la que se origina en la etapa embrionaria por una fusión incompleta de los procesos nasales mediales y procesos maxilares, representando uno de los defectos de nacimiento más frecuentes, generando una fisura en la zona labial semejante morfológicamente con la región labial que presentan los mamíferos lagomorfos de la familia Leporidae conocidos habitualmente como liebres, siendo el significado literal del apelativo labio leporino "labio de liebre".

\section{Réplica / Reply}

Sr. Editor: Los Dres. Mario Cantín e Iván Suazo G. llaman la atención sobre el uso del término "labio leporino". Opinan que debiera ser reemplazado por "labio fisurado", de acuerdo con el Comité Federal sobre Terminología Anatómica
Actualmente, en conformidad con los objetivos determinados por el Comité Federal sobre Terminología Anatómica (FCAT) y la Federación Internacional de Asociaciones de Anatomistas (IFAA) ${ }^{3,4}$ quienes buscan un correcto uso del lenguaje científico para toda la comunidad biomédica ibero-americana, el término labio leporino debería ser modificado por "labio fisurado", el cual describe de manera concreta este defecto del desarrollo evitando una comparación morfológica que en cierto modo se torna despectiva y no obedece a un lenguaje médico y científico adecuado.

Mario Cantín ${ }^{1,2}$, Iván Suazo Galdames ${ }^{1,2}$ ${ }^{1}$ Morfología, Departamento de Ciencias Básicas y Biomédicas, Universidad de Talca, Chile.

${ }^{2}$ Sociedad Chilena de Anatomía.

\section{Referencias}

1. Nazer J, Ramírez MC, Cifuentes L. 38 Años de vigilancia epidemiológica de labio leporino y paladar hendido en la maternidad del Hospital Clínico de la Universidad de Chile. Rev Med Chile 2010; 138: 567-72.

2. Real Academia Española. Diccionario de la Lengua Española. 22a Ed. Madrid, Editorial Espasa Calpe S.A.; 2001.

3. International Anatomical Terminology. New York, Thieme Medical Publishers; 1998.

4. Cruz GR, Rodríguez TA, Prates JC, Losardo RJ, Prates NEVB. Simposio Ibero-latinoamericano de Terminología Morfológica. Características Generales. Int J Morphol 2010; 28: 643-6.

Correspondencia a:

Dr. Mario Cantín, Morfología, Departamento de Ciencias Básicas y

Biomédicas, Oficina 104, Avenida Lircay s/n, Universidad de Talca, Talca,

Chile. Teléfono: 056-71-201756. E-mail: mcantin@utalca.cl

(FCAT) y la Federación Internacional de Asociaciones de Anatomistas (IFAA), quienes "buscan un correcto uso del lenguaje científico". Este término, propuesto por anatomistas, resulta incompleto desde el punto de vista médico-clínico.

Debo precisar que el término "labio leporino" es ampliamente usado en los países de habla hispana. El ECLAMC al igual que el International 
Clearinghouse for Birth Defects Monitoring Systems, incluyen en el diagnóstico de labio leporino a la fisura del labio y a la fisura labio palatina y hablan de "labio leporino con o sin paladar hendido". El paladar hendido aislado tiene una etiopatogenia totalmente diferente a la de labio leporino.
Estoy de acuerdo en que la terminología debería ser cambiada y usar la de "fisura labial y/o labiopalatina" cuando nos referimos al labio leporino, pero el uso generalizado de él nos ha permitido entender a lo nos referimos. Esta es nuestra posición sobre el uso de término "labio leporino".

Dr. Julio Nazer $H$. 